\title{
Enzymuntersuchungen am isolierten Glomerulum der Rattenniere
}

\author{
Von \\ H. E. FRANZ \\ Aus der Medizinischen Universitäts-Poliklinik Freiburg (Direktor: Prof. Dr. med. H. Sarre)
}

(Der Schriftleitung zugegangen am 23. Dezember 1962)

\begin{abstract}
Es wird über zwei Methoden der Isolierung der Glomerula und der Bestimmung von Enzymaktivitäten in dieser Struktur bei normalen und nierenkranken Ratten berichtet. Ein Vergleich zwischen Nierentinde und -mark wird angestellt. Die Bedeutung der Ergebnisse wird diskutiert und die durch die Methode bedingten Fehlermöglichkeiten aufgezeigt. Auf die Wichtigkeit die Untersuchungen nur an anatomisch genau definierbaren Strukturen des Nephrons vorzunehmen, wird ausdrücklich hingewiesen.
\end{abstract}

Two methods are described for the isolation of the glomerula from normal rats and tats with kidney disfunction, and for the determination of the activities of some constituent enzymes. The kidney cortex and medulla were compared. The significance of the results is discussed and possible sources of error are indicated. The importance of studying only anatomically well defined structures of the nephron is stressed.

Über die biochemischen Vorgänge in der Niere ist heute so gut wie nichts bekannt. Suchen wir in einem Lehrbuch der Biochemie nach Nierenspezifischen biochemischen Prozessen, so werden wir enttäuscht. Stoffwechselvorgänge, die sich ausschließlich in der Niere abspielen, sind bis heute unbekannt. Wie kann man sich erklären, $\mathrm{da} B$ die Niere mit denselben Enzymen wie sie in der Leber vorkommen so unerhört spezialisierte Transportaufgaben zu leisten vermag? Wir können darauf heute noch keine Antwort geben, weil unsere Methoden zur Erforschung des Stoffwechsels der Nierenzellen viel zu grob und undifferenziert sind. Die Unzulänglichkeit der Analysenmethode geht aus folgendem Vergleich hervor: Analysieren wir den Leberstoffwechsel mit Hilfe von Leberschnitten oder Leberhomogenaten, so dürfen wir das Resultat als ziemlich repräsentativ für die „Leberzelle" betrachten. Ganz anders ist es im Falle der Niere, bei der wir in enger räumlicher Anordnung ein Konglomerat langgezogener und ineinander verschlungener Funktionseinheiten, die Nephrone, vorfinden. Auch für den Biochemiker gilt, was Oliver (1) den Physiologen kritisch vorwarf: "There is no 'kidney', either structural or functional in chronic renal disease. The only useful or meaningful purpose of the word is to designate a mass" of tissue which, except in topographical, anatomic or surgical problems, has no significance until we analyze its constituents. And then it vanishes into the disparity of thousands of fantastically altered organs of strange design and anomalous behaviour ... Liquidate the 'kidney' and entitle the next symposium 'The Nephrons in Health and Disease'." - Dies gilt in gleicher Weise auch für die gesunde Niere. Die Verwendung von Nierenhomogenaten oder -schnitten wird daher höchstens in ausgewählten Fällen eine biochemische Analyse der Nierenfunktion ermöglichen (1a). Es ist daher auch für den Biochemiker zu fordern, daß er seine Untersuchungen nicht wie bisher mit der Gesamtniere ausführt, sondern sich auf bestimmte Abschnitte oder gar Zellen des Nephrons beschränkt. Diese Erwägu̇ngen haben in letzter Zeit dazu geführt, einen morphologisch leicht identifizierbaren Bestandteil des Nephrons, das Glomerulum, vom übrigen Nierengewebe abzutrennen und durch Aktivitätsbestimmung bestimmter Enzyme zu einem Einblick in seinen Stoffwechsel zu gelangen. In der vorliegenden Arbeit soll aufgezeigt werden, welche Methoden zur Isolierung der Glomerula zur Verfügung stehen und inwieweit die an ihnen durchgeführten Untersuchungen zum Verständnis ihres Stoff-" wechsels beigetragen haben.

$$
\text { - }
$$

\section{Metbodik der Isolierung von Glomerulum ais Rattennieren}

Solomon (2) und Mitarbeitern gelang es 1949 bei Versuchen, ein spezifisches Antiserum gegen Rattennierenglomerula zur Erzeugung von Masugi-Nephritis herzustellen, größere Mengen von Glomerula mechanisch von der übrigen Nierenrinde abzutrennen. Ähnliche Versuche sind seither wiederholt unternommen worden. Die Nachteile dieser Methoden bestanden jedoch darin, daß sie zu lange Zeit in Anspruch nehmen (Gefahr der Inaktivierung), daß eine grobe Veruntreinigung durch tubuläre Elemente auftritt oder daß bei der Isolierung Schwermetalle verwendet wurden (3).

Lowerl (4) konnte daher auch in den isolierten Glomerula keine Enzymaktivität nachweisen; nur BARKLEY und SINGH (5) machten einzelne Angaben über das Vorkommen von Enzymen, verwendeten aber keine standardisierten Bestimmungsmethoden. Lediglich zwei Methoden der Isolierung haben sich bewährt und hinreichend reproduzierbare Ergebnisse gebracht. Die erste, von Lowry (6) entwickelte Methode bedient sich der Mikrodissektion zur Isolierung: 
Vom oberen und unteren Pol der Niere werden mit einer gekühlten Rasicrklinge kleine Gewebsblöcke abgeschnitten und in flüssigem Stickstoff versenkt. Ohne aufzutauen werden von diesen Blöcken in einem Kryostaten bei $-21^{\circ} 28-30 \mu$ dicke Schnitte gemacht und diese lyophilisiert. Die Schnitte werden dann auf Zimmertemperatur gebracht und unter einem Dissektionsmikroskop die Glomerula mit einer feinen Stahlnadel aus dem Gewebe herausgetrennt. Die Glomerula werden dann auf einer empfindlichen Waage gewogen, homogenisiert und die Enzymaktivitäten nach von LowRY ausgearbeiteten Methoden bestimmt.

Der Nachteil dieser Methode besteht darin, daß es eines erheblichen apparativen Aufwandes bedarf und die Ausbeute an Glomerula gering ist. Atmungsversuche im Warburg-Apparat dürften deshalb kaum durchführbar sein; dagegen eignet sich die Methode vorzüglich für Untersuchungen an Nierenbiopsien, wie sie von Bonting et al. (7) durchgeführt wurden. - Bei der zweiten Methode handelt es sich um eine Modifizierung der von Solomon $(2,8,9)$ angegebenen Methode. Dabei werden die Glomerula aus der in kleinẹ Stücke geschnittenen Niere, nach vorheriger Abtrennung der Papillenspitze, durch Herauspressen in einem Homogenisator mit lose sitzendem Pistill abgetrennt. Die Säuberung von tubulären Elementen wird durch Zentrifugation gegen einen Saccharosegradienten und anschließend mehrfaches Sieben durch Nylonnetze mit bestimmter Maschenweite erreicht. Legt man den von KUNKEL (10) ermittelten Wert von 27000 Glomerula/g Rattenniere zugrunde, so kann man, wie Zählungen ergeben haben, $7 \%$ davon rein isolieren. Es genügen so die Nieren eines Tieres, um eine Anzahl Enzymaktivitäten zu bestimmen und Atmungsversuche durchzuführen.

\section{Morphologie}

Morphologische Untersuchungen der mit der Mikrodissektionsmethode isolierten Glomerula liegen nicht vor. Die Betrachtung der nach der Solomon-Methode isolierten Glomerula unter dem Mikroskop zeigt, da $B$ diese völlig von der Umgebung isoliert sind und daß sie nicht mehr an tubulären Elementen haften. Die Trennung der Glomerula vom übrigen Nierengewebe erfolgt meist im Bowmanschen Kapselraum; sie sind vom viszeralen Epithel umhüllt, das paritale Blatt fehlt. Nur etwa $5 \%$ aller Glomerula sind noch vom paritalen Blatt umhüllt. Der Gefäßstiel ist kurz und am Hilus des Glomerulum, abgerissen, enthält aber den juxtaglomerulären Apparat. Es besteht keine Tendenz zur Verklumpung, die Glomerula kleben jedoch leicht an Glasflächen.

\section{Enzymatische Untersucbungen an isolierten Glomerula}

Die isolierten Glomerula wurden bisher zu Untersuchungen der in ihnen enthaltenen Enzyme verwandt. Obschon die meisten Enzyme in der Zelle im Überschuß vorliegen und als Schrittmacher für den Stoffweechsel bedeutungslos sind, dürfen doch aus der quantitativen Verteilung der Enzyme in bestimmten Zellräumen Hinweise auf das Vorherrschen einzelner Stoffwechselwege erwartet werden. Um einen Ubberblick über den energetischen Stoffwechsel zu bekommen, wurden Enzyme der Glykolyse (Aldolase, Lactat-dehydrogenase (LDH)) und des oxydativen Stoffwechsels (Isocitronensäuredehydrogenase (ICDH), Malat-dehydrogenase (MDH), Glucose-6-phosphat-dehydrogenase (G-6-P-DH)) studiert.

$\mathrm{Zu}$ der von Dubach und Recant angewendeten Methodik der Enzymaktivitätsbestimmung verweisen wir auf ihre Arbeit (11). Zur Bestimmung der Enzymaktivitäten benützten wir folgende Methoden (vgl. 9):

1. Lactat-dehydrogenase (LDH): Optischer Test nach Kubowitz und Otr (12).

2. Glucose-6-phosphat-dehydrogenase (G-6-P-DH):Optischer Test nach WARBURg und Christian (13).

3. Isocitrat-dehydrogenase (ICDH): Optischer Test nach OCHOA (14).

4. Fructose-1,6-diphosphat-aldolase (ALD): Optischer Test nach RACKer (15).

5. Malat-dehydrogenase (MDH): Optischer Test nach Angaben in der "Test-Kombination Boehringer (TC-L; 15'981)“.

In der nachstehenden Tabelle (Tab. 1) sind die von den zwei Arbeitsgruppen gefundenen Resultate mit unseren verglichen. Die Werte von Dubach und Recant sowie BonTING und Mitarbeitern (16), die in Mol umgesetztes Substrat pro kg Gewebstrockengewicht/Std. angegeben sind, wurden unter der Annahme, daß $1 \mathrm{~g}$ Feuchtgewicht $=0,1 \mathrm{~g}$ Trockengewicht (Glomerulum) ist, in $\mu \mathrm{Mol}$ umgesetztes Substrat pro Stunde pro g Feuchtgewicht $\left(25^{\circ}\right)$ umgerechnet:

Tab. 1

Enzymaktivitäten in isoliertem Glomerula

\begin{tabular}{lcc}
\hline & Dubach/Recant & Richterich/Franz \\
\hline MDH & 3320 & 5700 \\
LDH & 2270 & 900 \\
ALD & - & 174 \\
ICDH & 520 & 248 \\
G-6-P-DH & 124 & $<1$ \\
\hline
\end{tabular}

Von Bontrg liegt nur die Aktivität der $\mathrm{LDH}$ vor, die einen Wert von 7410 ergab.

Obwohl die Absolutwerte der Enzymaktivitäten bei den zwei Gruppen beträchtliche Unterschiede aufweisen, so sieht man doch eine gute Übereinstimmung der Relativwerte (MDH hat die größte, G-6-P-DH die geringste Aktivität). Zum Vergleich der Enzymaktivitäten im isolierten Glomerulum seien die Enzymaktivitäten der Nierenrinde und der Papillenspitze hier angeführt (Tab. 2). Sie wurden berechnet als $\mu \mathrm{Mol} / \mathrm{Std}$./mg Eiweiß unter der Annahme, daß $1 \mathrm{~g}$ Feuchtgewicht $=0,2 \mathrm{~g}$ 
Eiweiß (Nierenrinde) bzw. $1 \mathrm{~g}$ Feuchtgewicht $=0,1 \mathrm{~g}$ Eiweiß (Papillenspitze) ist:

Tab. 2

Enzymaktivitäten der Nierenrinde und der Papillenspitze nach (9)

\begin{tabular}{lcc}
\hline & Nierenrinde & Papillenspitze \\
\hline LDH & 7680 & 3050 \\
ALD & 52 & 93 \\
MDH & 102000 & 15900 \\
ICDH & 608 & 435 \\
G-6-P-DH & 622 & 186 \\
\hline
\end{tabular}

Bevor man zu einer Interpretation dieser Befunde übergeht, sei auf einige, die Resultate beeinflussenden Fehlerquellen hingewiesen: Von großer Bedeutung ist zunächst die Reinheit und Intaktheit der isolierten Glomerula. Es muß sich die Frage erheben, ob die als glomeruläre Enzymaktivitäten gemessenen Werte nicht durch beigemischte Tubulusenzyme verfälscht werden. Es ist hierbei weniger an eine grobe Verunreinigung durch Tubulusstückchen in der glomerulären Fraktion gedacht, was bei der Mikrodissektionstechnik kaum in Frage kommt und bei der zweiten Methode sich durch sorgfältiges Sieben vermeiden läßt. Hingegen besteht die Möglichkeit, daß gelöste Enzyme an das Glomerulum absorbiert werden. Außerdem muß an die Möglichkeit eines Enzymverlustes aus den Glomerulumzellen bei der Manipulation während der Isolierung gedacht werden. Die Behandlung des Gewebes nach der Isolierung spielt gleichfalls eine große Rolle. Bei der Mikrodissektionsmethode wird das lyophilisierte Gewebe homogenisiert und in einem Mikroreagenzglas mit den Testsubstanzen zusammengebracht. Die mit der modifizierten SoLomon-Methode isolierten Glomerula wurden in 0,25 (isotoner) Saccharoselsg. homogenisiert und das Homogenat den in Puffer gelösten Substanzen zugesetzt. Bei beiden Methoden hat man keine Kontrolle aus welchen Zellräumen die Enzyme stammen. Aus dem Fehlen der Glutamatdehydrogenaseaktivität, die nach bisherigen Untersuchungen nur in Mitochondrien vorkommt, in unseren Homogenaten, schlossen wir, $\mathrm{da} ß$ die Mitochondrienenzyme bei unserer Aufarbeitung nicht in Lösung gehen. Im Gesamtnierenhomogenat haben Bontivg et al. (16) untersucht, aus welchen Räumen die Aktivität der $\mathrm{LDH}$ stammt. Sie trennten mit der in der Biochemie üblichen Methode der Differentialzentrifugation Zellkerne, Mitochondrien, Mikrosomen und übriges Zytoplasma (supernatant fraction). Dabei fand sich über $90 \%$ der LDH-Aktivität in der Zytoplasmafraktion, womit der Fehler bei Mitanalysieren der anderen Zellraumenzyme klein ist. Viel größer wird der Fehler jedoch bei Enzymen, wie der $\mathrm{ICDH}$ und $\mathrm{MDH}$, die als Komponenten des KrebsZyklus in hoher Konzentration in Mitochondrien jedoch auch im Zytoplasma mit noch unbekannter Funktion vorkommen. Die geforderte Auftrennung in die verschiedenen Zellräume ist, wie leicht ersichtlich, bei den wenigen durch Mikrodissektion gewonnenen Glome- rula sehr schwer, bei der zweiten Methode mit größerer Ausbeute an Glomerula leichter erreichbar. Schließlich $\mathrm{mu} ß$ noch der Beobachtung Rechnung getragen werden, daß LDH $(17,18,19,20)$, MDH $(21,22,23,24)$, ICDH $(21,22,25)$ und alkalische Phosphatase (26) heterogen sind, d. h. in verschiedenen molekularen Spezies vorkommen. So konnten WIELAND und PFLEIDERER etstmals nachweisen, daß die LDH des tierischen Gewebes sich in mehrexe Enzymspezies, sogenannte Isoenzyme, auftrennen läßt, die sich in ihrer Kinetik, Hemmbarkeit und elektrophoretischen Wanderung unterscheiden $(18,19,27)$. Im Nierenhomogenat konnten sie mittels Hochspannungselektrophorese fünf Komponenten mit LDH-Aktivität unterschieden $\left(\alpha_{1}, \alpha_{2}, \beta, \gamma_{1}\right.$ und $\gamma_{2}{ }^{-}$ Enzyme nach der gleichen Wanderungsgeschwindigkeit mit den entsprechenden Globulinen genannt).

Durch eine Trennung der Niere in Rinde, Papillenspitze und Glomerula sowie anschließender elektrophoretischer Auftrennung des.Gewebseiweißes wurden von RICHTERICH et al. (28) die Verteilung der fünf LDH-Isoenzyme in diesen Geweben bestimmt. Während in den kortikalen Tubuli das schnellwandernde sogenannte $\alpha_{1}$-Isoenzym vorherrscht, findet man in der Papillenspitze vorwiegend das langsamwandernde $\gamma$ Isoenzym. In den Glomerula findet man je $40 \%$ des $\alpha_{1}$ und $\gamma$-Enzyms. Es ist möglich, daß ein Teil der $\alpha_{1}{ }^{-}$ Aktivität durch beigemischtes tubuläres Enzym bedingt ist. Zur Deutung dieser Befunde ist die Beobachtung Pfleiderers und Wachsmutrs (29) bemerkenswert, die in ausschließlich unter aeroben Bedingungen funktionierenden Organen (Gehirn und Herzmuskel) überwiegend $\alpha_{1}$-Aktivität fanden. Dagegen überwog in Geweben, die auch unter anaeroben Bedingungen arbeiten (Skelettmuskel, Leber, Epidermis), das $\gamma$-Enzym. Diese Vethältnisse treffen auch auf die Niere zu. Aus Atmungsversuchen ist bekannt, daß die Nierenrinde eine hohe Sauerstoffaufnahme besitzt. Das Nierenmark dagegen gehört zu den intensivst glykolysierenden Geweben. Eigene Untersuchungen am isolierten Glomerulum (30) zeigten, daß auch diese Struktur über eine hohe Glykolyse verfügt. Dies zeigt im Zusammenhang mit dem großen Gehalt an $\gamma$-Enzym an, daß die oben beschriebene Gesetzmäßigkeit wahrscheinlich auch auf das Glomerulum zutrifft.

Für künftige Enzymaktivitätsuntersuchungen an isolierten Nierensubstraten wird es notwendig sein, die Extraktionsmethode zu standardisieren und zu versuchen neben den Zytoplasmaenzymen auch die der Mitochondrien zu erfassen. Bei den in mehreren molekularen Spezies vorkommenden Enzymen müssen die optimalen Reaktionsbedingungen für die einzelnen Enzyme gesucht werden. Es muß abschließend hervorgehoben werden, da $\beta$ allein die glomeruläre Fraktion annähernd homogen ist und überwiegend aus viszeralen Epithelzellen und Kapillarendothelien besteht. In der Papillenspitze sind histologisch mindestens drei Zelltypen, in der glomerulafreien Nierenrinde wahrscheinlich über zwanzig verschiedene Zelltypen zu unterscheiden. 
Enzy'mmuster der Nierenrinde, der Papillenspitze und der Glomerula

Seit einigen Jahren gelingt es durch den Nachweis organ-spezifischer Enzyme, etwa Enzymen der Harnstoffsynthese bei der Leber, auch mit biochemischen Methoden eine Organdiagnose zu stellen $(31,32)$. Ein wesentlicher Teil der heutigen klinischen „Enzymdiagnostik" beruht auf dem Nachweis solcher organspezifischer Enzyme im Serum. Seit einigen Jahren gelingt es BücHER und Mitarbeitern $(21,22,23)$ diese quantitative Betrachtungsweise auf die Enzyme des Energiestoffwechsels zu übertragen und Unterschiede in den Wegen der Energieproduktion zwischen den verschiedenen Organen aufzudecken. Abgesehen von einigen Resultaten aus der Histochemie fehlten bisher Angaben über das Enzymmuster der Niere, obschon gerade bei diesem Organ mit den seit Jahrzehnten bekannten Unterschieden des Energiestoffwechsels zwischen Nierenrinde und Mark interessante Ergebnisse zu erwarten sind. Tatsächlich konnten wir (9) unter Berücksichtigung der oben ausgeführten Einwände der angewandten Methodik beträchtliche Unterschiede in der Verteilung einiger Hauptkettenenzyme des CRaumes in den Glomerula, der Papillenspitze und Nierenrinde nachweisen. Das Auffinden hoher Konzentrationen von Malat- und Isocitrat-dehydrogenase, also Enzyme des oxydativen Stoffwechsels in der Nierenrinde, stimmen gut mit den Beobachtungen überein, da $\beta$ diese von allen Säugetierorganen die höchste Sauerstoffaufnahme aufweist (34). Physiologische Untersuchungen zeigten, $\mathrm{da} \beta$ sich in diesem Nierenabschnitt vor allem Resorptions- und Sekretionsvorgänge abspielen. Es ist wahrscheinlich, daß die dazu benötigte Energie aus dem oxydativen Stoffwechsel stammt, doch sind zahlreiche Fragen noch ungeklärt. Im Nierenmark stimmt die relativ hohe Aldolase- und LDH-Konzentration gut mit der von GyörGY beobachteten hohen Glykolyse überein (35). Die Unterschiede in Enzymprofil zwischen Nierenrinde und Mark lassen vermuten, daß der Ionentransport in diesen beiden Organteilen energetisch verschieden gekoppelt ist. In den Glomerula finden sich Verhältnisse, die sowohl von der Nierentinde als auch vom Nierenmark abweichen. Hier fällt die extreme Verminderung der Glucose-6-phosphat-dehydrogenaseAktivität bei gleichzeitiger Verminderung der DPNabhängigen Dehydrogenasen und gesteigerten Aldolaseaktivität auf. Eine Interpretation dieses extremen Enzymmusters ist zur Zeit nicht möglich.

\section{Untersucbungen bei Aminonucleosidnepbrose und Masugi- Nepbritis der Ratte}

Es lag nahe, die im gesunden Gewebe durchgeführten Untersuchungen auch auf Nierenerkrankungen mit vorwiegend glomerulärer Beteiligung auszudehnen. DUBACH und RECANT (11) bestimmten Enzymaktivitäten in isolierten Glomerula bei der sogenannten Aminonucleosidnephrose (Tab. 3). Sie fanden bei dieser experimentellen Nephrose, die einer etwa sechstägigen Vor- behandlung bis zum Eintreten der Proteinurie bedarf, erst Änderungen der Enzymaktivitäten mit beginnender Proteinurie.

Tab. 3

Fermentaktivitäten im normalen und aminonucleosidnephrotischem Glomerulum der Ratte nach (11)

\begin{tabular}{lccc}
\hline \multicolumn{1}{c}{ Ferment } & Normal & Nephrotisch & $P$ \\
\hline Alk. P'tase & $\left.3,07 \pm 0,33^{1}\right)$ & $2,24 \pm 0,30$ & $<0,01$ \\
HK & $0,534 \pm 0,028$ & $0,690 \pm 0,078$ & n. s. \\
G-6-P-DH & $1,190 \pm 0,098$ & $1,600 \pm 0,084$ & $<0,005$ \\
LDH & $26,6 \pm 3,0$ & $32,2 \pm 2,4$ & $<0,05$ \\
MDH & $32,0 \pm 1,7$ & $40,3 \pm 2,5$ & $<0,01$ \\
ICDH & $4,92 \pm 0,49$ & $5,59 \pm 0,61$ & n.s.
\end{tabular}

(In beiden Gruppen wurden je 6 Tiere untersucht. Die Aktivität ist ausgedrückt in Mol Substrat umgesetzt $/ \mathrm{kg}$ Trockengewicht $/ \mathrm{Std}$. bei $37^{\circ}$.)

1) Mittelwert \pm Standardfehler des Mittelwertes. $P=$ Wahrscheinlichkeit. n. s. = statistisch nicht signifikant verschieden.

Die Resultate weisen nach Ansicht der Autoren auf einen relativ aktiven oxydativen Stoffwechsel (MDH und ICDH) hin, dem ein weniger aktiver glykolytischer (HK und LDH) gegenübersteht. Der Hexose-monophosphat-Shunt, gemessen an der G-6-P-DH-Aktivität scheint ebenso von geringer metabolischer Bedeutung zu sein. Es bestand eine gute Korrelation zwischen Ausmaß der Enzymaktivitätsänderung und Schweregrad der Proteinurie. Die Ergebnisse bei der Masugi-Nephritis, bei der nur alkalische Phosphatase und G-6-P-DH im isolierten Glomerulum untersucht wurde, ergaben ähnliche Verhältnisse (36), (Tab. 4 und 5):

Tab. 4

Alkalische Phosphatase-Aktivität im Glomerulum bei MasugiNephritis (Mol/kg Trockengewicht/Std. bei $37^{\circ}$ )

\begin{tabular}{rcccc}
\hline Tag & Versuchstiere & $\begin{array}{c}\text { Anzahl } \\
\text { Ratten }\end{array}$ & Kontrollen & $\begin{array}{c}\text { Anzahl } \\
\text { Ratten }\end{array}$ \\
\hline 1. Tag & $2,033 \pm 0,417$ & 6 & $2,945 \pm 0,510$ & 3 \\
5. Tag & $1,816 \pm 0,351$ & 5 & $3,073 \pm 0,206$ & 3 \\
12. 'Tag & 1,468 & 1 & - & -
\end{tabular}

(Mittelwert aller Kontrolltiere: $3,006 \pm 0,289 \mathrm{Mol} / \mathrm{kg} / \mathrm{Std}$.)

Tab. 5

Glomeruläre G-6-P-DH-Aktivität bei Masugi-Neplritis (Mol/kg Trockengewicht/Std. bei $37^{\circ}$ )

\begin{tabular}{rcccc}
\hline Tag & Versuchstiere & $\begin{array}{c}\text { Anzahl } \\
\text { Ratten }\end{array}$ & Kontrollen & $\begin{array}{c}\text { Anzahl } \\
\text { Ratten }\end{array}$ \\
\hline 1. Tag & $1,802 \pm 0,070$ & 6 & $1,293 \pm 0,076$ & 3 \\
5. Tag & $1,836 \pm 0,051$ & 5 & $1,265 \pm 0,013$ & 3 \\
12. Tag & 1,473 & 1 & - & -
\end{tabular}

(Mittelwert aller Kontrolltierc: $1,278 \pm 0,024 \mathrm{Mol} / \mathrm{kg} / \mathrm{Std}$.) 
Das Auftreten von Enzymaktivitätsänderungen erst nach Einsetzen der Proteinurie und die Abhängigkeit des Ausmaßes von der Eiweißausscheidung lassen darauf schließen, daß diese Aktivitätsänderungen Folge des durch die massive Proteinurie veränderten Stoffwechsels sind. Es handelt sich dabei unseres Erachtens also um einen Sekundäreffekt. Die Hoffnung, durch diese Untersuchungen die primären zur Nephrose führenden Stoffwechselalterationen aufzufinden, erfüllten sich nicht.

\section{Literatur}

1. Oliver, J., J. Urol, Baltimore,63, 373 (1950). - 1a. SARre,H. u. H. Eiger, Z. Klin. Med. 136, 96, (1939) - 2. Solomon, D. H., J. W. Gardella, H. Fanger, F. M. Dethier und J. W. Ferrebee, J. Exper. Med. 90, 267 (1949). - 3. Cook, W. F. und G. IV. Pickering, Nature (London) 182, 1103 (1958). - 4. Lowell, D. I., S. A. Greenspon, C. A. Crakower und I. A. Bain, Amer. J. Physiol. 172, 709 (1953). - 5. BarkLEX, J. A. und I. D. Singh, Acta med. Scand. 154, 484 (1956). - 6. Lowry, O. H., J. biol. Chemistry 140, 183 (1941). - 7. Pollak, V. E., S. L. Bonting, R. C. MuehrCKe und R. M. Kark, J. Clin. Invest. 39, 1394 (1960). - 8. Richterich, R. und H. E. Franz, Nature (London) 188, 448 (1960). - 9. Richterich, R. und H. E. FraNZ, Biochem. Z. 334, 149 (1961). - 10. Kunker, P. A., Bull. John Hopkins Hosp. 47, 285 (1930). - 11. DuBACH, U. C. und L.ReCANT, J. Clin. Invest. 39, 1364 (1960). - 12. Kubowrtz, F. und P. OTr, Biochem. Z. 314, 94 (1943). - 13. Warburg, O. und W. ChrISTIAN, Biochem. Z. 287, 291, 440 (1936). - 14. OсноA, S., J. biol. Chemistry 174, 133 (1948). - 15. RACKER, E., J. biol. Chemistry 167, 843 (1947). - 16. Bonting, S. L., V. E. Pollax, R. C. MuEHRCKe und R. M. KARK, J. Clin. Invest. 39, 1381 (1960). 17. Wieland, T. und G. Pfleiderer, Angew. Chem. 69, 199 (1957). - 18. Wieland, T., G. Pfleiderer, I. Haupt und W. WÖRNER, Biochem. Z. 332, 1 (1959). - 19. WIELAND, T., G.
Pfleiderer und F. Ortandert, Biochem. Z. 331, 103 (1959). 20. WremE, R. J., Studies on Agar Gel Elektrophoresis. Brüssel, Arscia Uitgaven (1959). - 21. Bücher, T. und M. KLINGENBERG, Angew. Chem. 70, 552 (1958). - 22. Delbrück, A., H. SCHIMASSEK, K. BARTSCH und T. BüCher, Biochem. Z. 331, 297 (1959). - 23. Носевоом, G. H., J. biol. Chemistry 172, 619 (1948). 24. Shepherd, J. A. und G. Kalnitzky, J. biol. Chemistry 207, 605 (1954). - 25. Christie, G. S. und J. D. Judah, Proc. roy. Soc. B 141, 420 (1953). - 26. Gryder, R. M., J. S. Friedenwald und C. CarLson, Arch. Biochemistry 54, 281 (1955). - 27. Wieland, T. und G. Pfleiderer, Biochem. Z. 329, 112 (1957). 28. Richterich, R., P. Schafroth und H. E. Franz, Enzymol. biol. clin. 1, 114 (1961/62): - 29. Pfleiderer, G. und E. D. Wachsmure, Klin. Wschr. 39, 352 (1961). - 30. Franz, H. E. und R. Richterich, in Vorbereitung. - 31. RICHTERICH, R., Enzympathologie, Enzyme in Klinik und Forschung. Berlin, Göttingen, Heidelberg, Springer (1958). - 32. Richterich, R., Helvet. med. acta 26, 887 (1959). - 33. DeLbRÜCK, A., E. Z EsBE und T. Bücher, Biochem. Z. 331, 273 (1959). - 34. WARBURG, O., Über den Stoffwechsel der Tumoren. Berlin, Göttingen, Heidelberg, Springer. - 35. GyörgY, P., W. KELLER und T. BrehME, Biochem. Z. 200, 336 (1928). - 36. Dubach, U. C., L. Recant, J. Clin. Invest. 39, 981 (1960).

Dr. H. E. Franz

Medizinische Universitäts-Polikiinik 78 Freiburg i. Br., Hermann-Herder-Str. 6

\section{Zur Besprechung eingegangene Bücher}

Enzyme.im Blutplasma. Von Dr. B. Hess. VIII, 142 S., 33 Abb., 13 Tab., Gr.-8, DM 29,70.

Georg Thieme Verlag, Stuttgart 1962.

Ionenaustauscher. Eigenschaften und Anwendungen. Von K. DorfNer. $58 \mathrm{Abb}$. und $27 \mathrm{Tab}$. VII, $163 \mathrm{~S}$., DM 28,-. Walter de Gruyter \& Co., Berlin 1963.

Tritium-Markierung. Darstellung, Messung und Anwendung nach Wilzbach ${ }^{3} \mathrm{H}$-markierter Verbindungen. Von $\mathrm{M}$. WeNzEL und P. E. Schulze. $62 \mathrm{Abb}$. und 42 Tab. XII, 176 S., DM 26,Walter de Gruyter \& Co., Berlin 1962.

Lehrbuch der physiologischen Chemie.

Begründet von S. EDLBACHER. Von F. Leuthardt. 15., neubearbeitete und erweiterte Aufl. 76 Abb. XVI, 917 S., DM 42,-. Walter de Gruyter \& Co., Berlin 1963.
Mikromethoden für das klinisch-chemische und biochemische Labotatorium. Von H. MATTENHEIMER. $23 \mathrm{Abb}$. XI, $146 \mathrm{~S}$., DM 16,-. Walter de Gruyter \& Co., Berlin 1961.

Praktische Geburtshilfe für Studierende und Ärzte. Von W. Pschyrembel. 9., neubearbeitete und ergänzte Aufl. 477, teils mehrfarbige Abb. XVI, 652 S., DM 30,-. Walter de Gruyter \& Co., Berlin 1963.

Hormonbehandlung in der Dermatologie. Von K. WrinkLer. 55 Abb. XVI, 281 S., DM 46,-.

Walter de Gruyter \& Co., Berlin 1962.

Ratte und Maus. Versuchstiere in der Forschung.

Von E. HagemanN unter Mitarbeit von G. Schmidt. $75 \mathrm{Abb}$. und 198 Tab. X, 318 S., DM 48,-.

Walter de Gruyter \& Co., Berlin 1960.

Die Wiedergabe von Gebraucbsmamen, Handelsnamen, Warenbezeicbnungen und dgl. in dieser Zeitscbrift berecbligt nicbl zu der Annabme, daß solcbe Namen obne weileres von jedermann benützt werden dürften. Vielmebr bandelt es sicb bäufig um gesetzlicb gescbützte Warenzeicben, aucb wemn sie nicbt eigens als solcbe gekennzeicbnet sind.

Verlag Walter de Gruyter \& Co., vormals G. J. Göschen'sche Verlagshandlung - J. Guttentag, Verlagsbuchhandlung - Geotg Reimer - Karl J. Trübner - Veit \& Comp. 1 Berlin 30, Genthiner Str. 13; 1963. - Printed in Germany. - Satz und Druck: Walter de Gruyter \& Co., 1 Berlin 30, Genthiner Str. 13 ; - Anzeigen : Merkur-Werbung, Dr. K. Jeserich KG, 7 Stuttgart, 1, Postfach 740, Tel. 246358/59/50. - Für den Anzeigenteil verantwortlich : Willibald Plitzko. 